\title{
Tritium-Labeled Compounds VI. Alditols-1-t and Alditols-2- $t$
}

\author{
Harriet L. Frush, Horace S. Isbell, and Alexander J. Fatiadi
}

(June 1, 1960)

\begin{abstract}
By reduction of aldoses and aldonic lactones with lithium borohydride- $t$, the following 1-tritium-labeled alditols were prepared: D-arabinitol-1-t, D-lyxitol-1-t, D-ribitol-1- $t$, D-xylitol1-t, D-galactitol-1- $t$, D-mannitol-1 $(6)-t$, D-glucitol-1-t, D-talitol-1- $t$, L-gulitol-1- $t$ (D-glucitol $6-t)$, L-rhamnitol-1-t, D-glycero-D-gulo-heptitol-1-t, and 4-O- $\beta$-D-galactopyranosyl-D-glucitol$1-t$. By reduction of ketoses with lithium borohydride- $t$, the following epimeric pairs of 2-labeled alditols were prepared and subsequently separated: D-mannitol- $2(5)-t$ and $\mathrm{D}$ glucitol-2- $t$; L-gulitol-2-t (D-glucitol-5-t) and L-iditol-2- $t$; D-galactitol-2- $t$ and D-talitol-2- $t$; D-glycero-D-gulo-heptitol-2-t and D-glycero-D-ido-heptitol-2-t; and D-glycero-D-galacto-heptitol2-t and D-glycero-D-talo-heptitol-2-t.

The yields of the epimeric alditols formed from ketoses were determined by an isotopedilution technique. Stereomeric relationships are discussed for the labeled alditols and for the ketoses derivable from them by oxidation with Acetobacter suboxydans.
\end{abstract}

\section{Introduction and Discussion}

Tritium-labeled alditols are useful intermediates for synthesizing tritium-labeled ketoses and for studying a wide variety of chemical and biological reactions. As part of a program on the development of methods for synthesizing tritium-labeled carbohydrates $[1,2,3,4,5,6],{ }^{2}$ procedures have now been developed for preparing alditols position-labeled with tritium.

Nonradioactive sodium borohydride has been used for reducing aldoses [7] and lactones [8,9] to alditols. However, for the preparation of tritium-labeled materials, it was considered advantageous to use lithium borohydride- $t$ (instead of the sodium analog*), because it may be more easily prepared. ${ }^{3}$ Hence, processes were developed for the use of lithium borohydride- $t$ in preparing tritium-labeled alditols.

The experimental conditions under which lithium borohydride is used are critical, insofar as the extent and efficiency of the reduction are concerned. In the previous preparation of aldoses- $1-t$ by the reduction of aldonic lactones [4], lithium borohydride- $t$, dissolved in anhydrous pyridine, was added to a solution of the lactone in water. The use of pyridine as a solvent avoids decomposition of the hydride and appears to suppress the further reduction of the aldose to the alditol. However, in the preparation of labeled alditols, better yields were obtained when tetrahydrofuran, instead pyridine, was used as the solvent.

\footnotetext{
1 Part of a project on the development of met hods for the synthesis of radioactive carbohydrates, sponsored by the Division of Research of the Atomic Energy Commission. The tritium-labeled products described may be purchased from the National Bureau of Standards at a price of $\$ 10$ per 100 microcuries.

${ }^{2}$ Figures in brackets indicate the literature references at the end of this paper

3 These materials can be prepared by tritium-h ydrogen exchange, which occur at about $200^{\circ} \mathrm{C}$ for lithium borohydride and at about $350^{\circ} \mathrm{C}$ for sodium boro-
} hydride [10]. The preparation of lithium borohydride- $t$ is described in ref [3].
Alditols mav be prepared by the reduction of aldoses, aldonic lactones, or ketoses. Aldoses and lactones, on reduction, form alditols having, respectively, one and two atoms of hydrogen- $t$ at $\mathrm{C} 1$. Thus, the product derived from the lactone has twice the specific activity of that derived from the aldose. Ketoses, on reduction, form epimeric pairs of alditols having one atom of hydrogen-t at $\mathrm{C} 2$; subsequent separation of the alditols is necessary.

Tables 1,2, and 3 summarize the results obtained by the reduction of aldoses, aldonic lactones, and ketoses, respectively, to alditols. Yields of the alditols were determined by (a) radioactivity assay of the purified solutions and (b) isotope-dilution techniques. Table 3 gives the yields of the epimeric alditols formed from several ketoses, a subject of considerable theoretical interest.

Tritium-labeled products having high activities are subject to decomposition from self-radiation, and hence should not be held long in storage. The activities of the products listed in tables 1,2 , and 3 are adequate for most purposes, and decomposition over the course of several months has been slight. Position-labeled products of higher activity can be made, but these must be used within a relatively short time.

TABLE 1. Reduction of aldoses with lithium borohydride-ta

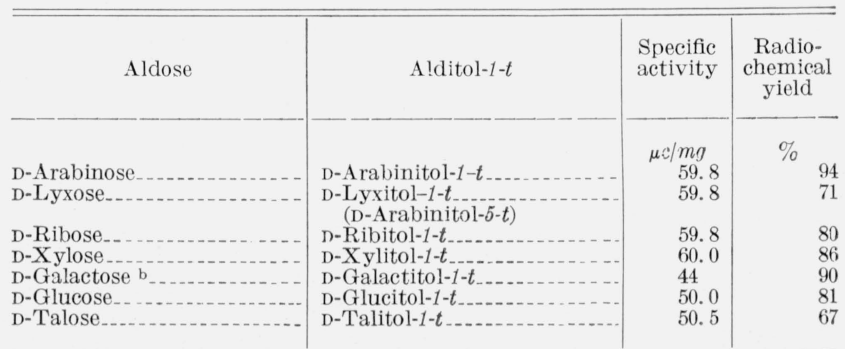

a Experimental details are given in section 4.1.

b Preparation reported earlier in ref [3]. The lithium borohydride had a specific activity different from that of the reductant used in other preparations. 
TABLE 2. Reduction of aldonic lactones with lithium borohydride-t a

\begin{tabular}{|c|c|c|}
\hline Lactone & Alditol- $1-t^{b}$ & $\begin{array}{l}\text { Radio- } \\
\text { chemical } \\
\text { yield }\end{array}$ \\
\hline $\begin{array}{l}\text { D-Arabono- } \gamma-1 \\
\text { D-X ylono- } \gamma-1-\end{array}$ & $\begin{array}{l}\text { D-Arabinitol-1-t } \\
\text { D-Xylitol-1-t }\end{array}$ & $\begin{array}{c}\% \\
79.2 \\
80.0\end{array}$ \\
\hline 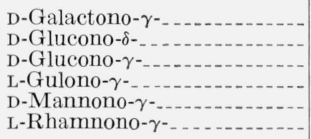 & $\begin{array}{l}\text { D-Galactitol-1-t } \\
\text { D-Glucitol-1- } t \\
\text { D-Glucitol- } 1 \text { - } t \\
\text { L-Gulitol-1- } t \text { (D-glucitol- } 6-t) \\
\text { D-Mannitol- } 1(6)-t c \\
\text { L-Rhamnitol- } 1-t\end{array}$ & $\begin{array}{l}71.5 \\
70.8 \\
70.3 \\
66.6 \\
76.6 \\
75.5\end{array}$ \\
\hline D-glycero-D-gulo-Heptono- $\gamma$-.-. & D-glycero-D-gulo-Heptitol-1-t_... & 67.4 \\
\hline Lactobiono- $\gamma-$ & 4-O- $\boldsymbol{\beta}$-D-Galactopyranosyl-D-glucitol-1- $t_{-}$ & 61.3 \\
\hline
\end{tabular}

a Experimental details are given in section 4.2 .

The products had an activity of approximately 19 millicuries per millimole

c The preparation of D-mannitol-1(6)- $t$ from 2,3:5,6-di- $O$-isopropylidene-Dmannofuranose was described in an earlier publication [3].

TABLE 3. Reduction of ketoses with lithium borohydride-t a

\begin{tabular}{|c|c|c|}
\hline Ketose & Alditol-2- $t$ & $\begin{array}{l}\text { Radio- } \\
\text { chemical } \\
\text { yieldb }\end{array}$ \\
\hline D-Fructose .. & $\begin{array}{l}\text { D-Mannitol-2(5)-t } \\
\text { D-Glucitol-2-t }\end{array}$ & $\begin{array}{c}\% \\
43.2 \\
42.1\end{array}$ \\
\hline L-Sorbose & $\begin{array}{l}\text { L-Gulitol-2-t } \\
\text { L-Iditol-2 } 2(5)-t_{t}\end{array}$ & 27.9 \\
\hline D-Tagatose.... & $\begin{array}{l}\text { D-Galactitol-2-t } \\
\text { D-Talitol-2-t }\end{array}$ & $\begin{array}{l}22.9 \\
59.5\end{array}$ \\
\hline D-gluco-Heptulose_. & $\begin{array}{l}\text { D-glycero-D-gluco-Heptitol-2-t } \\
\text { D-glycero-D-ido-Heptitol-2-t. }\end{array}$ & $\begin{array}{l}22.6 \\
32.1\end{array}$ \\
\hline D-manno-Heptulose. & $\begin{array}{l}\text { D-glycero-D-galacto-Heptitol-2-t } \\
\text { D-glycero-D-talo-Heptitol-2- } t\end{array}$ & $\begin{array}{l}33.2 \\
60.0\end{array}$ \\
\hline
\end{tabular}

a Experimental details are given in section 4.3

b Yields were determined by isotope dilution.

\section{Nomenclature of Position-Labeled Alditols and Related Ketoses}

The presence of an isotopic atom at a definite position in the molecule of an alditol gives rise to certain problems of nomenclature. An alditol that has no axis or plane of symmetry is related to two aldoses. If this alditol is position-labeled, the position of the label is designated differently in the two names. For example, the alditol obtained by reducing Dglucose-1- $C^{14}$ may be named either D-glucitol-1- $C^{14}$ or L-gulitol- $6-C^{14}$.

An unlabeled alditol that has a plane of symmetry is a meso compound derivable from either the $\mathrm{D}$ or the $\mathrm{L}$ form of an unlabeled aldose. However, this alditol is truly asymmetric if position-labeled, and is classified as D or L according to the configuration and the position of the label. Thus, D-galactitol-1- $t$ is enantiomorphic with L-galactitol- $1-t$, but is identical with L-galactitol-6- $t$; similarly, D-xylitol-1- $C^{14}$ may also be named L-xylitol- $5-C^{14}$.

An alditol that has an axis of symmetry is related to only one aldose. Because the two parts of the molecule are identical, the corresponding atoms or groups are indistinguishable. For instance, in Dmannitol, the structure and configuration are the same at $\mathrm{C} 1$ and $\mathrm{C} 6$ (as well as at $\mathrm{C} 2$ and $\mathrm{C} 5$, and at
C3 and C4). Hence, if the alditol is labeled in one position of the molecule, it is labeled also in the corresponding position. Thus, for example, the alditol obtained by reducing D-mannose- $1-C^{14}$ is D-mannitol$1(6)-C^{14}$.

Certain of the alditols are oxidized to ketoses by Acetobacter suboxydans. This organism oxidizes a compound containing the structure $\mathrm{HOH}_{2} \mathrm{C}-\mathrm{C}-\mathrm{C}-\mathrm{C}$

by converting the group at the penultimate carbon atom to -C- [11]. Table 4 lists the labeled ketoses<smiles>[CH]=O</smiles>

that can be produced by $A$. suboxydans from 1-tritium-labeled alditols having six or fewer carbon atoms. D-Fructose-1 (6)- $t$ and L-sorbose- 6 - $t$ have already been prepared. The latter sugar is an intermediate for the preparation of L-ascorbic- $6-t$ acid.

\section{Apparatus and Materials}

The reductions were conducted in a closed system, in 50-ml flasks each having a rubber-capped side-arm for the injection of liquids. The flasks were attached to a vacuum manifold, which was part of the equipment previously described for collecting and handling tritium gas [3].

Lithium borohydride- $t$ was prepared from nonradioactive lithium borohydride by hydrogen-tritium exchange. Solutions of lithium borohydride- $t$ in dry tetrahydrofuran were then prepared by the procedure given in ref [3].

TABLE 4. Tritium-labeled alditols and ketoses derivable from aldoses a

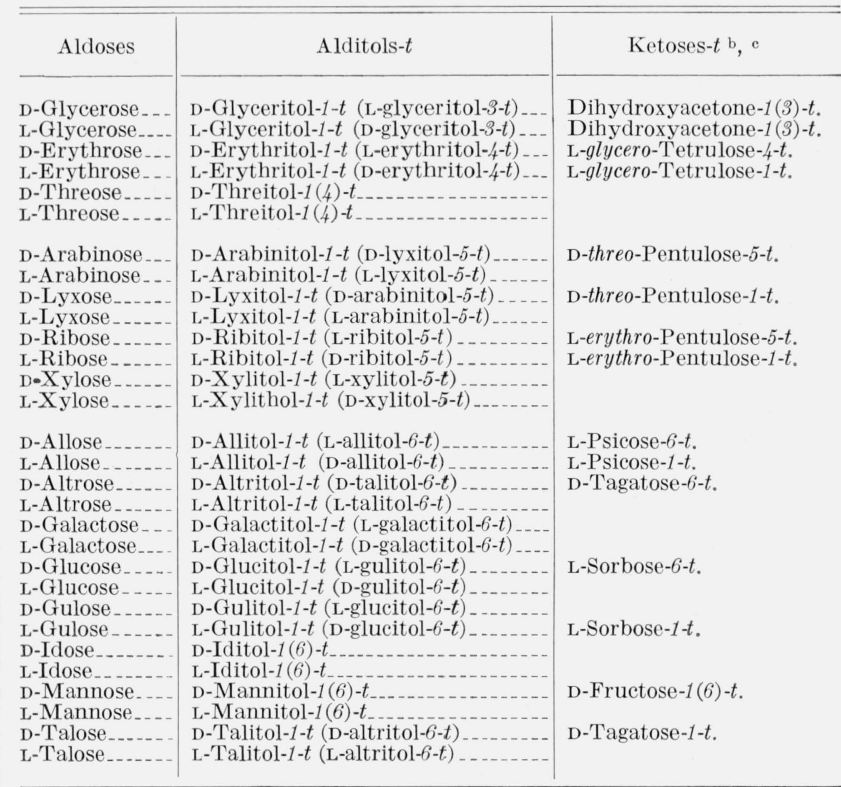

a Relationships are illustrated for compounds having six or fewer carbon atoms. b Ketoses derivable from sterically suitable alditols, through oxidation by Acetobacter suboxydans. The other alditols listed do not have the requisite configuration for attack by A. suboxydans.

figuration for attack by $A$. suboxydans.
c Systematic names are as follows: L-ribo-hexulose(L-psicose); D-lyxo-hexulose (D-tagatose); L-xylo-hexulose(L-sorbose); and D-arabino-hexulose (D-fructose).
(D) 
Radioactivity assays were made with a $2 \pi$, windowless, gas-flow, proportional counter. Materials with low activity were counted in films of sodium $O$ (carboxymethyl)cellulose on 2-in. flanchets [2]. Those with high activity were assayed as solutions in formamide. The procedure was essentially the same as that developed for the assay of carbon-14 [12], but was standardized with a sample having known tritium content. The counting efficiency is extremely low, but the precision of the method is excellent. Under the conditions used, one count per second is equivalent to $0.128 \mu \mathrm{c}$ of tritium.

\section{Procedures}

\subsection{Preparation of Alditols-1-t From Aldoses}

A magnetic stirring bar and 4 millimoles of the aldose to be reduced were placed in a $50-\mathrm{ml}$ reaction flask equipped with a rubber-capped side-arm. The flask was attached to the vacuum manifold and evacuated. The connection of the reaction flask to the manifold was closed, and the flask was cooled in a shallow ice-bath resting on a magnetic stirrer.

Five milliliters of ice-water containing 1 millimole (106 mg) of sodium carbonate was injected through the rubber cap by means of a hypodermic needle and syringe. The stirrer was started, and $2 \mathrm{ml}$ of a solution containing 1.0 millimole $(22 \mathrm{mg})$ of lithium borohydride- $t$ in dry tetrahydrofuran was added by needle through the rubber cap; stirring was continued for 15 minutes. The solution was allowed to stand at room temperature for several hours (or overnight) and was then frozen in liquid nitrogen. The by-product hydrogen- $t$, formed by reaction of the lithium borohydride- $t$ with water, was transferred to the manifold and either stored in a flask or converted to water-t. Finally, the connection to the manifold was closed and the flask was removed. The solvent (water and tetrahydrofuran) was evaporated in a rotary vacuum still equipped with a trap immersed in a dry-ice freezing bath. Water was added and the solution was again concentrated in the still; addition of water and concentration were repeated several times. Ultimately, the distillate in the trap was discarded as radioactive waste. The residue in the flask was dissolved in water, and the solution was passed through a column containing 10 $\mathrm{ml}$ of a cation-exchange resin. The effluent was evaporated to about $1 \mathrm{ml}$ in the vacuum still; then, about $15 \mathrm{ml}$ of methanol was added, and the solvent was again evaporated. Addition of methanol and evaporation were repeated several times in order to remove all boric acid as methyl borate. An aqueous solution of the residue was passed through a small column of mixed cation- and anion-exchange resins, and the effluent ${ }^{4}$ was concentrated under reduced pressure. The residue was crystallized from hot ethanol or other suitable solvent, and the specific activity of the product was determined as described in section 3 and ref [2]. The alditol-1-t was recrystallized until the specific activity became constant.

\subsection{Preparation of Alditols-1-t From Aldonic Lactones}

The procedure for preparing 1-tritium-labeled alditols from aldonic lactones was the same as that described in section 4.1, except for the following changes: (a) 2 millimoles of the aldonic lactone were reduced in place of 4 millimoles of the aldose; (b) sodium carbonate was omitted in the reduction step; ${ }^{5}$ and (c) 1.25 millimoles of lithium borohydride- $t$ were used in place of 1 millimole.

\subsection{Preparation of Alditols-2-t From Ketoses}

The method for reducing ketoses with lithium borohydride- $t$ was essentially the same as that given in section 4.1 for reducing aldoses. Two millimoles of the ketose were reduced with 0.5 millimole of lithium borohydride- $t$ having an activity of approximately 9 me per milliatom of hydrogen. The product was then treated as follows:

(a) The yields of the separate epimeric alditols-2-t were determined by an isotope-dilution technique. Aliquots of the solution containing approximately $5 \mu \mathrm{c}$ of tritium were diluted with $100 \mathrm{mg}$ of the nonradioactive alditol under investigation. The alditol carrier was then recrystallized repeatedly from a suitable solvent, ordinarily ethanol, until a product of constant activity was obtained. From the relative size of the aliquot used, the weight of the carrier taken, and the specific activity of the carrier after recrystallization, the total activity of the alditol in the parent solution was readily calculated.

(b) After removal of aliquots for analysis, the solution was concentrated and the epimeric alditols were separated by fractional crystallization, usually from ethanol. In most cases, one of the alditols crystallized more readily than the other; satisfactory separations were obtained by seeding the sirup with one epimer and removing the crystals of the substance before crystals of the other appeared. In some cases, the products were separated by addition of the nonradioactive alditol as carrier. The identity and purity of the alditol-2- $t$ were confirmed by the following isotope-dilution technique:

A 1-mg sample of the purified alditol-2-t of known radioactivity was diluted with $100 \mathrm{mg}$ of the pure, nonradioactive alditol. The carrier mixture was recrystallized three times, and the product was assayed for radioactivity. If $\mathrm{A}$ and $\mathrm{B}$ were, respectively, the specific activities of the alditol-2- $t$ and the carrier mixture, then the purity of the alditol$2-t$ (in percent) was $(101 \mathrm{~B} / \mathrm{A}) \times 100$. All of the alditols reported in table 3 gave results within 4 percent of the expected value.

Appreciation is expressed to Joseph D. Moyer and Lorna T. Sniegoski, who conducted some of the laboratory work reported.

\footnotetext{
${ }^{4}$ The effluent, when tested with a conductivity meter, showed the absence of ionic impurities.

${ }_{5}^{5}$ Reduction of aldonic lactones to alditols is a two-step process, in the first of which the aldose is formed. Although an alkaline medium facilitates reduction of the aldose to the alditol, alkali tends to convert the lactone to the nonreducible salt. Hence, it was omitted in this reduction.
} 


\section{References}

[1] NBS Tech. News Bul. 43, 130 (1959); Chem. and Eng. News 37, No. 39, 101 (1959).

[2] H. S. Isbell, H. L. Frush, and R. A. Peterson, J. Research NBS 63A, 171 (1959).

[3] H. S. Isbell and J. D. Moyer, J. Research NBS 63A, 177 (1959).

[4] H. S. Isbell, H. L. Frush, and N. B. Holt, J. Research NBS 64A, 177 (1960).

[5] H. S. Isbell, H. L. Frush, and J. D. Moyer, J. Research NBS 64A, 359 (1960).

[6] H. S. Isbell, H. L. Frush, and N. B. Holt, J. Research NBS 64A, 363 (1960).
[7] M. Abdel-Akher, J. K. Hamilton, and F. Smith, J. Am. Chem, Soc. 73, 4691 (1951).

[8] M. L. Wolfrom and H. B. Wood, J. Am. Chem. Soc. 73, 2933 (1951); M. L. Wolfrom and K. Anno, J. Am. Chem. Soc. 74, 5583 (1952).

[9] H. L. Frush and H. S. Isbell, J. Am. Chem. Soc, 78, 2844 (1956).

[10] W. G. Brown, L. Kaplan, and K. E. Wilzbach, J. Am. Chem. Soc. 74, 1343 (1952).

[11] R. M. Hann, E. B. Tilden, and C. S. Hudson, J. Am. Chem. Soc. 60, 1201 (1938).

[12] A. Schwebel, H. S. Isbell, and J. D. Moyer, J. Research NBS 53, 221 (1954) RP2537.

(Paper 64A5-67) 


\section{Publications of the National Bureau of Standards}

\section{Selected Abstracts}

Electric polarizability of a short right circular conducting cylinder, T. T. Taylor, J. Research NBS 64B, No. 3, 135 (1960). A method similar to that employed by Smythe for calculating the capacitance of a freely charged short right circular conducting cylinder is used to calculate the electric polarizability tensor in the principal axis system for such a cylinder. Calculations to an accuracy of approximately five significant figures are carried out for cylinders with radius to half-length ratios of $1 / 4,1 / 2,1,2$, and 4 . The results are applicable to the design of artifical dielectrics.

Split Runge-Kutta method for simultaneous equations, J. R. Rice, J. Research NBS 64B, No. 3, 151 (1960).

Consider two simultaneous first order differential equations $x^{\prime}(t)=F(x, y, t), y^{\prime}(t)=G(x, y, t)$. Runge-Kutta type integration methods are developed which allow different integration steps to be used for these equations. These methods retain the desirable properties of Runge-Kutta methods, namely the self-starting property and ease of change of integration step. Two different approaches are considered and extensive experimental work is reported upon. Experiments are done both in situations where these methods are advantageous and where they are not. It is seen that these methods are more efficient than the normal Runge-Kutta methods if they are at all applicable and in ideal situations they give the same accuracy with $90 \%$ less computation. These methods are applicable to six degree of freedom missile simulations, for which they were developed.

A reduction formula for partitioned matrices, E. V. Haynsworth, J. Research NBS 64B, No. 3, 171 (1980).

A theorem of L. Goddard and H. Schneider, concerning square matrices $A$ and $B$, of orders $n$ and $m$ respectively, which satisfy an equation $A X=X B$ for some $n \times m$ matrix $X$, is generalized here for rectangular matrices $A$ and $B$, with dimensions $n_{1} \times n_{2}, m_{1} \times m_{2}$, which satisfy $A X_{2}=X_{1} B$, where $X_{i}$ has dimensions $n_{i} \times m_{i}$ for $i=1,2$. This result is used to find reduction formulae for partitioned matrices with submatrices, $A_{i j}$, having dimensions $n_{i} \times n_{j}$, and satisfying equations $A_{i j} X_{j}=X_{i} B_{i j}$. The reduction formulae given here are also generalizations of a theorem by J. Williamson concerning partitioned matrices whose submatrices are all square and satisfy $A X=X B$, where $B$ is triangular and $X$ is square.

Phase angle master standard for 400 cycles per second, J. H. Park and H. N. Cones, J. Research NBS 64C, No. 3, $229(1960)$

A continuously variable, 0 to 180 degree, phase shift standard for $400 \mathrm{c} / \mathrm{s}$ is described in detail. It consists of a $\pi$-section line made up of twelve $14.6^{\circ}$ and three $4.3^{\circ}$ sections to provide for two sizes of coarse steps and an $\mathrm{RC}$ circuit at the input to the line to provide for fine steps and a continuous fine control. A method for accurately adjusting the characteristic impedance of all $\pi$-sections to the same value, which is used as the termination, was devised. Under these conditions it is shown that the phase shift introduced by each $\pi$-section can be accurately computed from a measured value of inductance. The phase shift of each $\pi$-section was also determined by an experimental procedure dependent upon a $180^{\circ}$ phase shift introduced by a toroidal transformer. The values obtained by these two independent methods agree to within 0.01 degree.

Disturbances due to the motion of a cylinder in a two-layer liquid system, L. H. Carpenter and G. H. Keulegan, $J$. Research NBS 64C, No. 3, 241 (1960).

The disturbance created at the interface of a two-layer liquid system by the horizontal motion of a cylinder in the upper layer is studied for various sizes and shapes of cylinders, depths of the liquids, cylinder velocities, and density ratios. The disturbances fall into three categories. First, when the layers are of equal thickness, in most cases a train of progressive oscillatory waves is produced at the interface. Second, when the depth of the denser layer is much less than the depth of the fresh-water layer, the profile of the interface usually resembles that corresponding to positive internal solitary waves. Third, when the depth of the denser layer is much greater than the depth of the fresh-water layer, in most cases an internal hydraulic jump is produced. The characteristics of the disturbances in each category are related to the size of the cylinder, the depths of the liquids, the cylinder velocity, the density ratio, and the total distance of travel of the cylinder. A theoretical analysis is given for disturbances of the first category.

Microwave absorption in compressed oxygen, A. A. Maryott and G. Birnbaum, J. Chem. Phys. 32, No. 3, 686 (1960).

Precise measurements of the absorption in oxygen resulting from the small magnetic dipole moment are reported at frequencies near $2.3,9$, and $23 \mathrm{kMc}$ and at pressures in the range from 3 to 70 atoms. Up to 10 atmospheres the resonant contribution agrees with the Van Vleck-Weisskopf theory and the line width increases in proportion to the pressure. The line width (relaxation frequency) for the nonresonant contribution also increases in proportion to the pressure but is only about one-third as large as the resonant line width. Above 20 atmospheres the resonant absorption shows anomalous behavior resembling that previously noted in the case of the inversion spectra of $\mathrm{NH}_{3}$ and $\mathrm{ND}_{3}$. In particular, the resonant frequency appears to decrease rapidly while the line width changes much less rapidly than the pressure.

Studies of infrared absorption spectra of solids at high pressures, E. R. Lippincott, C. E. Weir, A. Van Valkenburg, and E. N. Bunting, Spectrochim. Acta 16, p. 58 (1960).

Infrared spectra of solids were studied with a diamond pressure cell in the wavelength range 5-15 $\mu$ at pressures between $1 \mathrm{~atm}$ and 50,000 atm. The calibration of the cell at the 14,000 atm transition of $\mathrm{NaNO}_{2}$ is described. Spectra were studied for aromatic organic compounds, inorganic hydrates, and ammonium halides. In general, band shifts produced by pressure were to higher frequencies and at most $10 \mathrm{~cm}^{-1} / 10,000 \mathrm{~atm}$. Many bands exhibited large changes in intensity. Occasionally bands increased in intensity or were unaffected but in general a decrease in intensity was observed at elevated pressure. Representative spectra are given, one to a pressure of $160,000 \mathrm{~atm}$. Suggestions for the causes of the frequency shifts are given.

Nickel-aluminum alloy coatings produced by electrodeposition and diffusion, D. E. Couch and J. H. Connor, J. Electrochem. Soc. 10\%, No. 4, 272 (1960).

Nickel-aluminum alloy coatings were produced by diffusion of aluminum electrodeposited over nickel. The aluminum was plated from baths operated at $25^{\circ}-1000^{\circ} \mathrm{C}$. The alloys were much harder than nickel and were superior to nickel coatings in salt spray, atmospheric exposure, and air oxidation tests. Attempts to codeposit the two metals were not successful.

Energy requirements of mechanical shear degradation in concentrated polymer solutions, A. B. Bestul, J. Chem. Phys. 32, No. 2, 350 (1960)

Molecular weight decrease by mechanical shearing results when solutions of around $10 \%$ of polyisobutene having average molecular weights above 500,000 are forced through a capillary at nominal rates of shear above $10,000 \mathrm{sec}^{-1}$. Comparison of observed plots of shear load $v s$ duration of shearing at fixed rates of shear during this degradation process with the corresponding estimated plots which would be expected to obtain if degradation did not occur provide a means of evaluating the amount of applied shearing energy 
which is dissipated by the degradation process. The result is several hundred thousand kilocalories per mole of broken bonds, which is several thousand times the bond energy of carbon-carbon bonds. This finding is consistent with the hypothesis that whenever a bond breaks the system loses much of the free energy temporarily stored in bonds and macromolecular chains located in a comparatively large volume surrounding the broken bond, these bonds and chains having been involved in concentrating the required activation energy into the ruptured bond.

Low-temperature transport properties of commercial metals and alloys. II. Aluminums, R. L. Powell, W. J. Hall, and H. M. Roder, J. Appl. Phys. 31, 496 (1960).

The thermal conductivity, electrical resistivity, Lorenz number, thermoelectric force, and thermoelectric power are given in the temperature range $4^{\circ}-120^{\circ} \mathrm{K}$ for ten aluminums and aluminum alloys: high-purity, 1100-F, 1100-O, 3003-F, 2024-T4, 5052-O, 5083-O, 5086-F, 5154-O, and 6063-T5. Four of the samples show a maximum in thermal conductivity, the others do not. For the four high-thermal conductivity samples the separate components in the electronic thermal resistivity are resolved; for the others, components in both the electronic and the lattice thermal resistivities are given. The residual electrical resistivities vary from $2.5 \times 10^{-8}$ to $3.2 \times 10^{-6}$ ohm-cm. The Lorenz numbers for the high-conductivity samples fall considerably below the Sommerfeld value $2.44 \times 10^{-8}$ watt-ohm $/{ }^{\circ} \mathrm{K}^{2}$; those for the low-conductivity samples are somewhat above the Sommerfeld value. The thermoelectric power of some of the alloys is positive with respect to the high-purity sample, for others it is negative. The various properties, methods of analysis, and separation of components are discussed in detail.

Low-temperature transport properties of commercial metals and alloys. III. Gold-cobalt, R. L. Powell, M. D. Bunch, and E. F. Gibson, J. Appl. Phys. 3, 504 (1960).

The thermal conductivity, electrical resistivity, and Lorenz number are given in the temperature range $4-100^{\circ} \mathrm{K}$ for the widely used thermocouple wire, gold-2.1 atomic percent cobalt alloy. The total thermal conductivity is relatively low: from 0.01 watt $/ \mathrm{cm}^{\circ} \mathrm{K}$ at $4^{\circ} \mathrm{K}$ to 0.23 at $100^{\circ} \mathrm{K}$. The lattice contribution to the total thermal conductivity is larger than the electronic contribution. The electrical resistivity is nearly independent of temperature, but does have a definite minimum of about $1.20 \times 10^{-5} \mathrm{ohm}-\mathrm{cm}$ at $30^{\circ} \mathrm{K}$. Because of the relatively large contribution of the lattice term to the total thermal conductivity, the Lorenz number is considerably above the electronic Sommerfeld value, $2.44 \times 10^{-8}$ watt-ohm/ ${ }^{\circ} \mathrm{K}^{2}$.

Infrared studies of dense forms of ice, E. R. Lippincott, C. E. Weir, and A. Van Valkenberg, Communication to Editor, J. Chem. Phys. 32, No. 3, 612 (1960).

Preliminary infrared studies in the water-ice system at high pressures have been conducted. Spectra in the $1 \mu-15 \mu$ range were obtained for liquid water, Ice I, and for ice at two elevated pressures at about $-27^{\circ} \mathrm{C}$. At $9,000 \mathrm{~atm}$ the run is in the Ice VI field and at 3,000 atm it is close to the II-III transition line. Although the exact phases cannot be specified because of uncertainties in equilibrium the spectra at elevated pressures differ markedly from Ice I. The details of the patterns are interpreted as indicating weak hydrogen bonding in dense ices with an $\mathrm{O}-\mathrm{H}$... O angle differing from $180^{\circ}$.

Several new methods to measure the thermal diffusivity of semiconductors, J. H. Becker, J. Appl. Phys. 31, No. 3, 612 (1960).

The techniques suggested in this note are well suited for measurements of thermal diffusivity on small semiconducting samples. The thermal diffusivity is derived from changes in the steady-state temperatures when the front surface of the specimen is heated at a sinusoidally time-varying rate (ac) and in some cases with a nontime-varying component (dc) The temperature changes are usually deduced from thermalelectric effects produced in the samples. These effects include the bolometric, Nernst, and Seebeck effects. The bolometric effect depends upon the variation of the electrical conductivity with temperature $(d \sigma / d T)$, while the Nernst and Seebeck effects depend upon the Nernst coefficient $Q_{n}$, and thermoelectric power $\theta$, respectively.

Correlation effects in impurity diffusion, J. R. Manning, Phys. Rev. 116, No. 4, 819 (1959).

Expressions are developed giving the correlation factor for an impurity diffusing in an otherwise pure lattice. These expressions will apply in general since, in the derivation, there is no requirement that there must be a large binding energy tending to create vacancy-impurity complexes. When this requirement is eliminated, accurate expressions can be obtained even for the nonclose packed lattices. Both vacancy and interstitialcy mechanisms are treated. A matrix method developed by LeClaire and Lidiard for the face-centered cubic lattice is applied to various lattices to obtain approximate expressions for the correlation factor. Then a correction is applied giving the effect of those dissociating vacancies (or interstitialcies) which return to the impurity. It is found that the effect is the same as if only a fraction of the dissociative jumps actually occurred. If it is assumed that the effect of an impurity is quite localized, the probability of return can be calculated in a very straightforward manner. It is shown that the effect of returning vacancies (or interstitialcies) can be quite important. The resulting expressions are used to estimate the correlation factor in nondilute alloys.

Cavity resonator dielectric measurements on rod samples, H. Bussey, Insulation, p. 26 (Nov. 1959).

Resonant cavity methods of measuring dielectric constant are especially valuable when the sample is in the shape of a rod. The rod is put on the axis of a circular cylindrical cavity. Some problems encountered in this method are the long and involved calculations, errors due to a hole in the cavity and plate for inserting a sample, the inconvenience of the necessary frequency changes when $\mathrm{TM}_{0 m 0}$ modes are used, and the difficulty of making accurate $Q$ measurements. Solutions to all of these problems are available at $9000 \mathrm{Mc}$, and to some extent at other microwave frequencies. Based on experimental data and theory, hole corrections have been obtained both for $\mathrm{TM}_{0 m 0}$ and $\mathrm{TE}_{011}$ modes. These corrections are very small for the $\mathrm{TE}_{011}$ mode, and since this mode is tuneable, a simple measuring system without frequency shifts can be set up, with an accurate micrometer moving the end plate. $Q$ measurements may be made with this micrometer too. Instead of measuring $Q$, however, the change in cavity transmission and a theoretical calculation of the change of iris coupling can be used to obtain the sample loss. The automatic computer is used to evaluate the raw data, or to form curves and tables to be used in making calculations. Measurements on sapphire rods have shown that the sensitivity for $\tan \delta$ is about $3 \times 10^{-5}$ at $9000 \mathrm{Mc}$.

A technique for reducing errors in permeability measurements with coils, B. L. Danielson and R. D. Harrington, Proc. IRE 48, No. 3, 365 (1960).

A method is described for eliminating the majority of the errors associated with permeability measurements using coils containing toroidal magnetic materials of rectangular cross section. Data is given to support the technique, and it is shown that correct answers can be obtained even for a small number of turns on the coil.

Low temperature phase transition of colemanite, A. Perloff and S. Block, Letter to Editor, Am. Mineralogist, p. 229 (1960). Precession photographs of the $h \mathrm{Ol}$ and $\mathrm{Okl}$ reciprocal lattice nets of colemanite $\left[\mathrm{CaB}_{3} \mathrm{O}_{4}(\mathrm{OH})_{3} \cdot \mathrm{H}_{2} \mathrm{O}\right]$ were taken at room temperature and at $-30^{\circ} \mathrm{C}$ using $\mathrm{Mo} \mathrm{K}_{\alpha}$ radiation with a $\mathrm{Zr}$ filter. Within the limits of error there was no change in the cell dimensions, which agree with the published values, and no changes were observed on the Okl net. However, the low temperature $h O l$ net contained very weak reflections from the $70 \overline{1}, 50 \overline{2}, 90 \overline{5}$, and $70 \overline{6}$ planes which establishes that the glide plane does not exist in the low temperature form. The transition between the room temperature $\mathrm{P} 2 / \mathrm{a}$ phase and the low temperature $\mathrm{P} 2_{1}$ phase is readily reversible. 
Magnetic properties of polycrystalline materials, D. M. Grimes, R. D. Harrington, and A. L. Rasmussen, J. Phys. Chem. Solids 12, 28 (1959).

The variation of the magnetic $Q$ with internal magnetization is discussed using both the domain rotation and the domainwall motion model of magnetization change. The variation of the reversible susceptibilities with magnetic moment is reported on four samples, and the results are compared with results from the frequency spectra in the initial and remanent states. The distribution of magnetic moments in the system as a function of angle between individual and averaged moments is discussed in terms of an infinite series expansion in Legendre polynomials. The coefficients of the first four terms can be measured. Experimental data are given for the first three.

Low angle X-ray diffraction of fibrous polyethylene, A. S. Posner, L. Mandelkern, C. R. Worthington, and A. F. Diorio, J. Appl. Phys. 31, No. 3, 536 (1960).

Highly axially oriented fibers of linear polyethylene are shown to display four orders of well defined, meridionally directed diffraction maxima corresponding to a fundamental spacing of $408 \mathrm{~A}$. An interpretation of the low angle X-ray pattern is given in terms of the fiber morphology and the Hess-Kiessig postulate as to the origin of the periodic variation in electron density. The relative macroscopic length of the fibers was systematically altered both by thermal treatment and by crosslinking, melting, and recrystallization. The magnitude of the spacings observed does not bear any direct relation to the change in length incurred, but reflects the change in crystallite size that develops because of annealing, partial melting or the introduction of crosslinks. It is also shown that in a completely shrunken fiber, where wideangle X-rav diffraction shows that the crystallites are randomly arranged relative to one another, discrete diffraction maxima are observed at about $255 \mathrm{~A}$. These maxima are, however, circular in shape. Intermediate types of line shapes are also observed, which depend solely on the crystallite orientation and not on the method by which the orientation is developed.

The source-function in a non-equilibrium atmosphere. IV. Evaluation and application of the net-radiative-bracket, R. N. Thomas, Astrophys. J. 131, 429 (1960).

The net rate of a radiative transition between two atomic energy levels depends upon the local radiation field through a factor which we define as the Net Radiative Bracket (NRB). The results of previous papers in this series are applied to show that NRB depends only upon the ratio of radiation absorbed in the line to the local value of the source function in the line and to evaluate this ratio as a function of optical depth in the line. These results may also be applied to the question of the local energy balance in a chromosphere-type atmosphere and to the problem of radiative stability.

The melting temperature of natural rubber networks, D. E. Roberts and L. Mandelkern, J. Am. Chem. Soc. 82, No. 5, 1091 (1960)

The isotropic melting temperatures, $T_{m}^{i}$, of natural rubber networks which were formed either by chemical reaction or by the action of ionizing irradiation were determined by dilatometric techniques. Substantial depression of the melting temperature with increasing amounts of crosslinking was observed for networks formed from randomly coiled chains irrespective of whe method by which the crosslinks were introduced. It is also found that the melting temperatures of networks formed from highly ordered chains are substantially greater than corresponding networks formed from random chains. The difference in melting temperatures for these two types of networks can be quantitatively attributed to the decrease in configurational entropy of the former type networks which occur as a consequence of the chain disposition at the time of their formation.

Ionic charges of glass surfaces and other materials, and their possible role in the coagulation of blood, D. Hubbard and G. L. Lucas, J. A ppl. Physiol. 15, No. 2, 265 (1960). Some observations on the physicochemical role played by glass surfaces in accelerating the "normal"coagulation rate of blood have been made, in light of the nonmigratable negative ionic charge which appears on leached glass surfaces and the orientation of the positively charged calcium ions of the blood at the fluid-glass interface. Numerous other materials, including artificial arteries, heart valves, bandages and "live" skin, have been investigated with respect to the ionic nature of their surfaces and their effect on blood coagulation. An effort was made to establish the ionic charge of the endothelial layer of the circulatory system; however, the procedure used in this investigation does not lend itself to in vivo or even convincingly to in vitro determinations. The ionic nature of the materials was determined from the uneven distribution of $\mathrm{Ag}\left(\mathrm{NH}_{3}\right)_{2}{ }^{+}$and $\mathrm{Br}^{-}$ions in full-strength aqueous ammonia, caused by the nonmigratable ionic charge characteristic of the surfaces. Such data appear to be qualitatively acceptable provided the material does not form any compound with either $\mathrm{Ag}\left(\mathrm{NH}_{3}\right)_{2}{ }^{+}$or $\mathrm{Br}^{-}$ions which is more insoluble than $\mathrm{AgBr}$, or does not have an isoelectric point between $p \mathrm{H} 7$ and $p \mathrm{H} 13.3$.

Emission spectra of $\mathrm{N}_{2}, \mathrm{O}_{2}$, and $\mathrm{NO}$ molecules trapped in solid matrices, H. P. Broida and M. Peyron, J. Chem. Phys. 32, No. 4, 1068 (1960).

Molecular systems are observed in emission in solid products from a gas discharge trapped at liquid helium temperature. Previous tentative molecular assignments have been checked with the help of isotopic substitutions of oxygen and nitrogen. The Herzberg system (A bands) of oxygen $\left(\mathrm{A}^{3} \Sigma_{\mu}{ }^{+}-\right.$ $\left.\mathrm{X}^{3} \Sigma_{g^{-}}\right)$is analyzed and the molecular constants are derived for a molecule trapped in a nitrogen matrix. Another system (M bands) is attributed to the NO molecule $\left({ }^{4} \pi-\mathrm{X}^{2} \pi\right)$.

Correction for systematic wavelength shifts in atomic beam devices, R. L. Barger and K. G. Kessler, J. Opt. Soc. Am. 50, No. 4, 352 (1960).

Light emitted or absorbed by atoms in an atomic beam is shifted in frequency relative to the frequency for a stationary atom if the light ray is not normal to the trajectory of the atom. When photoelectric measurements are made by observing, on the optical axis, the central spot of an interference pattern, this shift is caused by any non-orthogonality between the atomic beam axis and the optical axis. When an interference pattern is photographed, an additional shift is present which varies in magnitude along the radius of the pattern. The magnitude of the shift is calculated for each case, and devices which correct for this effect are described. These devices, consisting of a spherical mirror or a system of plane mirrors with a beam splitter, combine light from both sides of the atomic beam. This gives one positive and one negative shift which compensate to give the true frequency. For photoelectric recording of interference fringes, complete compensation can be achieved. In the case of photographic recording, it is shown that the residual relative frequency shift $\delta \nu / \nu$ is normally less than about one part in $10^{12}$.

Theory of the magnetic and spectroscopic properties of neptunium hexafluoride, J. C. Eisenstein and M. H. L. Pryce, Proc. Roy. Soc. A255, 181 (1960).

The magnetic properties and the optical absorption spectrum of $\mathrm{NpF}_{6}$ are interpreted on the basis of an appropriate model for the molecule. The theory, in its simplest form, is not in perfect accord with all the available data. Consequently various physical effects such as covalence, vibronic interaction, the coriolis force on the unpaired electron, and the John-Teller effect which might affect the agreement of theory and experiment are discussed in a qualitative or semi-quantitative way. It is pointed out that the $g$-value may vary with temperature so that one must be cautious when comparing the paramagnetic resonance and susceptibility data with theoretical predictions. Numerous experiments which would help to clarify the interpretation are suggested.

High pressure form of analcite and free energy change with pressure of analcite reactions, H. S. Yoder, Jr., and C. E. Weir, Am. J. Sci. 258-A, 420 (1960).

The compressibility of four natural analcites has been obtained up to 10,000 atm. at $25^{\circ}$ C. All specimens show abnormal compressibility and one exhibits a reversible tran- 
sition at about 8,400 atm. Abnormal increase of compressibility with pressure usually precedes a transition, and it is concluded that a similar transition lies at higher pressures than those investigated for the three remaining specimens. The compressibility and density data were used with previously obtained data to compute the volume change and free energy change with pressure for the reactions 2 analcite $\rightleftharpoons$ nepheline + albite +2 water, analcite $\rightleftharpoons$ jadeite + water, and analcite + quartz $\rightleftharpoons$ albite + water. The volume change for all the reactions is negative for all pressures investigated at $25^{\circ} \mathrm{C}$.

The increase in birefringence after compression in the analcite which exhibited the transition is attributed to either strain induced by the volume discontinuity or by retention of a possible lower svmmetry of the high-pressure form. Lower symmetry in a high-pressure form is not uncommon in previously investigated substances.

Class of nonlinear dielectric materials, P. H. Fang, R. S. Roth, and H. Johnson, J. Am. Ceram. Soc. 43, No. 3, 169 (1960).

A class of non-linear dielectric materials has been found, the members of which are formed by the reaction of a $\mathrm{BaNb}_{2} \mathrm{O}_{6}$ and a perovskite-type phase. At low temperature, dielectric hysteresis loops appear, similar to usual ferroelectric loops. Since one component of these new materials can be a transition element, it may be possible to find materials which are both ferroelectric and ferrimagnetic.

Bremsstrahlung linear polarization, J. W. Motz and R. C. Placious, Nuovo Cimento 15, Series X, $5 \% 1$ (1960).

This work presents a general quantitative description of the bremsstrahlung linear polarization on the basis of original experimental data and available theoretical calculations. The results give the dependence of the polarization on (a) the initial electron kinetic energy, $T_{0}$, in a range from $10^{-2}$ to $10^{3} \mathrm{Mev}$, (b) the photon energy in a range from $0.1 T_{0}$ to $T_{0}$, (c) the photon emission angle in a range from 0 to 180 degrees, and (d) the atomic number of the target in a range from 4 to 79 . The experimental data were obtained for a range of electron energies from 0.05 to $1.0-\mathrm{Mev}$ with beryllium, aluminum, and gold targets. Theoretical estimates of the polarization were calculated from the Sommerfeld-Kirkpatrick-Wiedmann results for the nonrelativistic energy region, the Olsen-Maximon results for the extreme relativistic energy region, and the Gluckstern-Hull (Born approximation) results for the intermediate energy region. Final results are expressed in terms of the peak polarization and the corresponding peak angle as a function of the electron and photon energies, and the most accurate estimates of the polarization are given on the basis of the combined experimental and theoretical data.

Chemically induced vibrational excitation: hydroxyl radical emission in the 1-3 micron region produced by the $\mathrm{H}+\mathrm{O}_{3}$ atomic flame, D. Garvin, H. P. Broida, and H. J. Kostkowski, J. Chem. Phys. 32, No. 3, 880 (1960).

A detailed investigation has been performed in the 1 - to $3-\mu$ wavelength region on the radiation emitted by the hydroxyl radical from a low-pressure flame of ozonized oxygen and atomized hydrogen. Wavelengths and photon intensities have been obtained for about 300 lines in the $\mathrm{OH}$ vibrationrotation bands $V-V-\Delta V$ where $\Delta V=3, V=9$ to 5 , and for $\Delta V=2, V=9$ to 2 . Relative photon band intensities have been determined from the overdetermined set of data by a method of successive approximations using an IBM 650 computer. Dipole moment parameters have been calculated using the above data and Morse oscillator transition probabilities.

Approximate rotational and vibrational Boltzmann distributions exist with an average rotational "temperature" of $560^{\circ} \mathrm{K}$ for the $P$ branches, $460^{\circ} \mathrm{K}$ for the $R$ and $Q$ branches, and a vibrational "temperature" of $9250^{\circ} \mathrm{K}$ for the $\Delta V=2$ and 3 bands. The absence of radiation from levels $V>9$ confirms the nonthermal character of the excitation and its dependence on the energetics of the reaction $\mathrm{O}_{3}+\mathrm{H} \rightarrow \mathrm{OH}+\mathrm{O}_{2}$.
Moreover, flux calculations based on the assumption that in this case collisional deactivation transition probabilities are proportional to the radiation probabilities show that there is an appreciable $\mathrm{OH}$ production in all levels $V<9$ and the main flux of $\mathrm{OH}$ through any population level is via $\Delta V=1$ transitions. Possible causes for the large changes in line intensity with pressure and smaller but significant changes with flows or concentrations are discussed.

Photographs of the high-altitude nuclear explosion "Teak," W. R. Steiger and S. Matsushita, J. Geophys. Research 65, No. 2, 545 (1960).

A sequence of four photographs of the August 1, 1958, highaltitude nuclear explosion "Teak" near Johnston Island in the Pacific is shown. These photographs were taken from Maui, Hawaii, at an altitude of 3050 meters and $1300 \mathrm{~km}$ from the explosion. Three main features of these photographs are evident and are discussed: an auroral arc directed southward; an expanding envelope; and an airglow cloud. The auroral arc extending southward from the explosion is interpreted as the glow produced by a stream of $\beta$-decay electrons directed along the earth's magnetic field. This arc apparently extended into the southern hemisphere and was observed from Apia, Samoa. The expanding envelope is interpreted as an excitation-recombination phenomenon produced by an expanding shock front. By assuming an average speed of propagation of $1.3 \mathrm{~km} / \mathrm{sec}$ of the shock front, the times at which the photographs were taken are estimated. The airglow cloud is interpreted as a residue of ionized material having a lifetime estimated at 15 to 30 minutes. Assuming an electron density of 10 times the normal $F_{2}$-region value, an effective recombination coefficient of 10 times the normal $F_{2}$ value is obtained. The airglow cloud ascended at a rate of approximately $1000 \mathrm{~m} / \mathrm{sec}$ and expanded horizontally at a rate of approximately $300 \mathrm{~m} / \mathrm{sec}$.

Appraisal of Land's work on two-primary color projections, D. B. Judd, J. Opt. Soc. Am. 50, No. 3, 254 (1960).

An analysis of the results of Land's experiments with twoprimary color projections has been carried out in terms of the known phenomena of object-color perception. It is shown that no new theory is required for the prediction of Land's result that two-primary color projections can produce object-color perceptions of all hues; nor for his result that many choices of pairs of primaries yield substantially the same object-color perceptions. Land's hypothesis that when the colors of the patches of light making up a scene are restricted to a one-dimensional variation of any sort, the observer usually perceives the objects in that scene as essentially without hue, is new; several special cases of it are supported by previous work as well as Land's. This hypothesis deserves the serious attention of research workers in object-color perception.

\section{Other NBS Publications}

Journal of Research, Section 64B, No. 3, July-September 1960. 75 cents.

Electric polarizability of a short right circular conducting cylinder. T. T. Taylor. (See above abstract.)

Distribution of quantiles in samples from a bivariate population. M. M. Siddiqui.

Split Runge-Kutta method for simultaneous equations. J. R. Rice. (See above abstract.)

A reduction formula for partitioned matrices. E. V. Haynsworth. (See above abstract.)

Selected bibliography of statistical literature, 1930 to 1957 : III. Limit theorems. L. S. Deming.

Journal of Research, Section 64C, No. 3, July-September 1960. 75 cents.

A new method of measuring gage blocks. J. B. Saunders.

Gage blocks of superior stability: initial developments in materials and measurement. M. R. Meyerson, T. R. Young, and W. R. Ney. 
Variation of resolving power and type of test pattern Francis E. Washer and William P. Tayman.

A multiple isolated-input network with common output. C. M. Allred and C. C. Cook.

Phase angle master standard for 400 cycles per second. J. H Park and H. N. Cones. (See above abstract.)

Disturbances due to the motion of a cylinder in a two-layer liquid system. L. H. Carpenter and G. H. Keulegan. (See above abstract.)

\section{Journal of Research, Section 64D, No. 5, September-October 1960. 70 cents.}

ELF electric fields from thunderstorms. A. D. Watt.

Field strength measurements in fresh water. G. S. Saran and G. Held.

Electrical resistivity studies on the Athabasca Glacier, Alberta, Canada. G. V. Keller and F. C. Frischknecht

Amplitude distribution for radio signals reflected by meteor trails. A. D. Wheelon.

Computation and measurement of the fading rate of moonreflected UHF signals. S. J. Fricker, R. P. Ingalls, W. C. Mason, M. L. Stone, and D. W. Swift.

On the theory of wave propagation through a concentrically stratified troposphere with a smooth profile. H. Bremmer.

Polarization and depression-angle dependence of radar terrain return. I. Katz and L. M. Spetner.

Methods of predicting the atmospheric bending of radio rays. B. R. Bean, G. D. Thayer, and B. A. Cahoon.

Loss in channel capacity resulting from starting delay in meteor-burst communication. G. R. Sugar.

Elementary considerations of the effects of multipath propagation in meteor-burst communication. G. R. Sugar, R. J. Carpenter, and G. R. Ochs.

Use of logarithmic frequency spacing in ionogram analysis. G. A. M. King.

Guiding of whistlers in a homogeneous medium. R. L. Smith

Propagation of microwaves through a magneto-plasma, and a possible method for determining the electron velocity distributions. A. L. Cullen.

On electromagnetic radiation in magneto-ionic media. Kogelnik.

Radiation and admittance of an insulated slotted-sphere antenna surrounded by a strongly ionized plasma sheath. J. W. Marini.

A contribution to the theory of corrugated guides. G. Piefke.

High-gain, very low side-lobe antenna with capability for beam slewing. A. C. Wilson.

Shielding of transient electromagnetic signals by a thin conducting sheet. N. R. Zitron.

Cylindrical antenna theory. R. H. Duncan and F. A. Hinchey.

Table of wavenumbers, 2000 A to 7000 A, C. D. Coleman W. R. Bozman, and W. F. Meggers, NBS Mono, 3, Vol. (1960) $\$ 6.00$.

Mercury barometers and manometers, W. G. Brombacher, D. P. Johnson, and J. L. Cross, NBS Mono. 8 (1960) 40 cents.

Bibliography on molecular and crystal structure models, D. K. Smith, NBS Mono. 14 (1960) 15 cents.

Calibration of line standards of length and measuring tapes at the National Bureau of Standards, L. V. Judson, NBS Mono. 15 (1960) 15 cents (supersedes NBS Circ. 572).

Quarterly radio noise data-March, April, May 1959, W. Q. Crichlow, C. A. Samson, R. T. Disney, and M. A. Jenkins, NBS Tech. Note 18-2 (PB151377-2) (1960) \$1.00.

Analog-digital conversion equipment for electrocardiographic data, L. Taback, NBS Tech. Note 42 (PB151401) (1960) $\$ 1.25$.

A summary of VHF and UHF tropospheric transmission loss data and their long-term variability. D. A. Williamson, V. L. Fuller, A. G. Longley, and P. L. Rice, NBS Tech. Note 43 (PB151402) (1960) \$2.25.

Tables for the statistical prediction of radio ray bending and elevation angle error using surface values of the refractive index, B. R. Bean, B. A. Cahoon and G. D. Thayer, NBS Tech. Note 44 (PB151403) (1960) 50 cents.

Operating instructions for AR $\mathrm{N}-2$ auxiliary log-linear noise recorder, R. T. Disney and C. A. Samson, NBS Tech. Note 45 (PB151404) (1960) 50 cents.

Report on the IGY oblique-incidence sporadic $E$ and $F$ scatter program, J. W. Finney and E. K. Smith, Jr., NBS Tech. Note 48 (PB151407) (1960) $\$ 2.50$

Dynamic measurements of the magnetoelastic properties of ferrites, V. E. Bottom, NBS Tech. Note 49 (PB151408) (1960) $\$ 1.00$.

Isotopic abundance ratios reported for reference samples stocked by the National Bureau of Standards, F. L. Mohler, NBS Tech. Note 51 (PB161552) (1960) 50 cents. Field strength calculations for E.L.F. radio waves, J. R. Wait and N. F. Carter, NBS Tech. Note 52 (PB161553) (1960) 50 cents.

Physical properties of synthetic-rubber-base dental impression materials, W. A. C. Miller, Jr., W. C. Hansen, G. Dickson, and W. T. Sweeney, J. Am. Dental Assoc. 60, 211 (1960)

The effect of multipath distortion on the choice of operating frequencies for high-frequency communication circuits, D. K. Bailey, IRE Trans. Ant. Prop. AP-y, No. 4, 397 (1959).

Liquid hydrogen from chemical and nuclear rockets, R. B. Scott, Discovery XXI, No. 2, 74 (1960)

Phosphorescence of nitrogen and nitrogen-argon deposited films at $4.2^{\circ}$ K, H. P. Broida and R. W. Nicholls, Letter to Editor J. Chem. Phys. 32, No. 2, 623 (1960).

Peculiarities of the ionosphere in the far east: A report on IGY observations of sporadic $E$ and $F$-region scatter, E. K. Smith, Jr., and J. W. Finney, J. Geophys. Research 65, 885 (1960).

Detecting radiation, L. Costrell, Chem. Eng. News, p. 132 (1960).

Opportunities in dental research, G. C. Paffenbarger, J. Am. Dental Assoc. 60, 413 (1960).

Departures of hydrogen from L.T.E. in a stellar atmosphere and the consequent structure of the solar chromosphere, S. R. Pottasch, Commun. Observ. Roy. Belgique No. 157, entitled The empirical determination of the stellar photospheric structure, Paper 11, 67 (1959).

Pilot plant data for hydrogen isotope distillation, T. M. Flynn, Chem. Eng. Progr. 56, No. 3, 37 (1960).

VLF phase characteristics deduced from atmospheric waveforms, A. G. Jean, W. L. Taylor, and J. R. Wait, J. Geophys. Research 65, 907 (1960).

Short-wave fadeouts without reported flares, H. DeMastus and M. Wood, J. Geophys. Research 65, No. 2, 609 (1960).

On the propagation of ELF radio waves and the influence of a nonhomogeneous ionosphere, J. R. Wait, J. Geophys, Research 65, No. 2, 597 (1960).

Precision Zeeman modulation microwave spectrometer, R. W. Zimmerer, Rev. Sci. Instr. 31, 106 (1960).

Chemistry, food, and civilization, A. T. MePherson, J. Wash. Acad. Sci. 50, No. 3, 1 (1960).

World maps of $F 2$ critical frequencies and maximum usable frequency factors for use in making ionospheric radio predictions, D. H. Zacharisen and V. Agy, J. Geophys. Research 65, 593 (1960)

Recent experimental evidence favouring the $p \mathrm{~K}_{1}(p)$ correlation function for describing the turbulence of refractivity in the troposphere and stratosphere, K. A. Norton, J. Atmospheric and Terrest. Phys. 15, 206 (1959).

Prediction of sunspot numbers for cycle 20, W. B. Chadwick, Nature, p. 1787 (1959).

The melting of crystalline polymers, L. Mandelkern, Rubber Chem. and Technol. XXXII, No. 5, 1392 (1959).,

Sampling of leather, J. Mandel and C. W. Mann, J. Sci. and Ind. Research 18A, No. 12, 575 (1959).

Atmospheric limitations on electronic distance measuring equipment, M. C. Thompson, Jr., H. B. Janes, and F. E. Freethey, J. Geophys. Research 65, 389 (1960).

Weighted restricted partitions, M. Newman, Acta Arith. V, 371 (1959).

A continuous poker game, A. J. Goldman and J. J. Stone, Duke Math. J. 27, No. 1, 41 (1960). 
Proposed specification for impression material; synthetic rubber base, dental, W. A. C. Miller, Jr., W. C. Hansen, G. Dickson, and W. T. Sweeney, J. Am. Dental Assoc. 60, 224 (1960).

Some results on the cross-capacitances per unit length of cylindrical three-terminal capacitors with thin dielectric films on their electrodes, D. G. Lampard and R. D. Cutkosky, Inst. Elec. Engrs. (London, England) Monograph No. 351M, 1 (1960).

A class of non-linear dielectric materials, P. H. Fang, R. S. Roth, and H. Johnson, J. Am. Ceram. Soc. 43, 169 (1960).

The linear viscoelastic behavior of rubberlike polymers and its molecular interpretation, R. S. Marvin, Viscoelasticity: Phenomenological aspects, p. 27 (Academic Press, New York, N.Y., 1960).

Low-frequency solar bursts and noise storms, A. Boischot, R. H. Lee, J. W. Warwick, Astrophys. J. 131, No. 1, 61 (1960).

Mass spectrometry, V. H. Dibeler and R. M. Reese, Anal. Chem. 32, No. 5, 211 (1960)

The components of power appearing in the harmonic analysis of a stationary process, M. M. Siddiqui, presented at Symp. on Statistical Methods in Radio Wave Propagation, Univ. California, Los Angeles, June 18-20, 1958, reprinted from Statistical methods in radio wave propagation (Pergamon Press, New York, N.Y., 1960).

Mechanical and electromechanical properties of indium antimonide, R. F. Potter, J. H. Wasilik, and R. B. Flippen, Ch. 12, Mechanical properties of intermetallic compounds, p. 265 (John Wiley \& Sons, Inc., New York, N.Y., 1960).

Electrometric pH determination, R. G. Bates, Chimia 14, 111 (1960).

Measurement of radiation exposure at the walls of medical $\mathrm{X}$-ray rooms for determining protective barrier requirements, S. W. Smith and J. R. Brooks, ATOMPRAXIS 6, No. 3, 77 (1960).

Introduction-Can you measure it? L. V. Judson, Am. Soc. Tool \& Mfgr. Engrs. Tech. Paper 239, Book 1, 60, $1(1960)$.

Some fundamentals of modern dimensional metrology, I. H. Fullmer, Am. Soc. Tool \& Mfgr. Engrs. Tech. Paper 240, Book 1, 60, 1 (1960).
The error in prediction of $F 2$ maximum usable frequencies by world maps based on sunspot number, E. L. Crow and D. H. Zacharisen, sec. II, Radio propagation phenomenology, in Statistical methods in radio wave propagation, p. 248 (Pergamon Press, New York, N.Y., 1960).

The use of $2,2,4,4,6,8,8$-heptamethylnonane as a primary diesel fuel, T. W. Mears, R. M. David, and F. L. Howard, ASTM Bul. No. 245, p. 75 (1960).

Sinn und bedeutung der strahlenschutznormen, L. S. Taylor, Rontgen-Blatter XIII, Heft 2, 33 (1960).

Geomagnetic disturbance and velocity of slow-drift solar radio bursts, M. B. Wood and C. S. Warwick, Nature 184, No. 4697, 1471 (1959).

Thermoelectric effects, H. P. R. Frederikse and W. W. Scanlon, ch. 7.6, Methods of experimental physies, Vol. VI, p. 114 (Academic Press, Inc., New York, N.Y., 1959).

The outlook for machine translation, F. L. Alt, Proc. Western Joint Computer Conf., Vol. 17, p. 203, San Francisco, Calif. (May 1960).

Further evidence of a solar corpuscular influence on largescale circulation at $300 \mathrm{Mb}, \mathrm{N}$. J. MacDonald and W. O. Roberts, J. Geophys. Research 65, No. 2, 529 (1960).

Method for measurement of $E^{\prime} / I^{\prime}$ in the reciprocity calibration of condenser microphones, W. Koidan, J. Acoust. Soc. Am. 32, No. 5, 611 (1960).

Microphone diaphragm null method for sound pressure measurements, W. Koidan, J. Acoust. Soc. Am. 32, No. 4 505 (1960).

An evaluation of a cesium beam frequency standard, R. C. Mockler, R. E. Beehler, J. A. Barnes, Symp. Quantum Electronics-Resonance Phenomena, p. 127 (Columbia University Press, New York, N.Y.) 1960.

Publications for which a price is indicated (except for NRS Technical Notes) are available only from the Superintendent of Documents, U.S. Government Printing Office, Washington 25, D.C. (foreign postage, one-fourth additional). Technical Notes are available only from the Office of Technical Services, U.S. Department of Commerce, Washington 25, D.C. (Order by $P B$ number.) Reprints from outside journals and the NBS Journal of Research may often be obtained directly from the authors. 